\title{
Regulation of the Escherichia coli HipBA Toxin-Antitoxin System by Proteolysis
}

\author{
Sonja Hansen ${ }^{1}$, Marin Vulić ${ }^{1}$, Jungki Min ${ }^{2}$, Tien-Jui Yen ${ }^{2}$, Maria A. Schumacher ${ }^{2}$, Richard G. Brennan ${ }^{2}$, \\ Kim Lewis ${ }^{1 *}$
}

1 Antimicrobial Discovery Center, Department of Biology, Northeastern University, Boston, Massachusetts, United States of America, 2 Department of Biochemistry, Duke University School of Medicine, Durham, North Carolina, United States of America

\begin{abstract}
Bacterial populations produce antibiotic-tolerant persister cells. A number of recent studies point to the involvement of toxin/antitoxin (TA) modules in persister formation. hipBA is a type II TA module that codes for the HipB antitoxin and the HipA toxin. HipA is an EF-Tu kinase, which causes protein synthesis inhibition and dormancy upon phosphorylation of its substrate. Antitoxins are labile proteins that are degraded by one of the cytosolic ATP-dependent proteases. We followed the rate of $\mathrm{HipB}$ degradation in different protease deficient strains and found that HipB was stabilized in a lon background. These findings were confirmed in an in vitro degradation assay, showing that Lon is the main protease responsible for HipB proteolysis. Moreover, we demonstrated that degradation of HipB is dependent on the presence of an unstructured carboxy-terminal stretch of HipB that encompasses the last 16 amino acid residues. Further, substitution of the conserved carboxy-terminal tryptophan of HipB to alanine or even the complete removal of this 16 residue fragment did not alter the affinity of HipB for hipBA operator DNA or for HipA indicating that the major role of this region of HipB is to control HipB degradation and hence HipA-mediated persistence.
\end{abstract}

Citation: Hansen S, Vulić M, Min J, Yen T-J, Schumacher MA, et al. (2012) Regulation of the Escherichia coli HipBA Toxin-Antitoxin System by Proteolysis. PLoS ONE 7(6): e39185. doi:10.1371/journal.pone.0039185

Editor: Valerie de Crécy-Lagard, University of Florida, United States of America

Received March 27, 2012; Accepted May 21, 2012; Published June 15, 2012

Copyright: (c) 2012 Hansen et al. This is an open-access article distributed under the terms of the Creative Commons Attribution License, which permits unrestricted use, distribution, and reproduction in any medium, provided the original author and source are credited.

Funding: This work was supported by National Institutes of Health grant R01 GM061162 (to KL), the Robert A. Welch Foundation grant G-0040 (to RGB), and MD Anderson Fellowship (to MAS). The funders had no role in study design, data collection and analysis, decision to publish, or preparation of the manuscript.

Competing Interests: The authors have declared that no competing interests exist.

*E-mail: k.lewis@neu.edu

\section{Introduction}

Bacterial populations stochastically produce a small number of non-growing persister cells that are tolerant to antibiotics [1-4]. Persisters are phenotypic variants that are genetically identical to the susceptible cells within a clonal population. Thus, persistence is a non-heritable, transient trait [5,6]. Previously, we have shown that the recalcitrance of biofilms is largely due to the presence of persisters $[7,8]$. Recent studies by our group link persistence to chronic infectious disease. In the case of cystic fibrosis patients infected with Pseudomonas aeruginosa, high persister (hip) mutants are selected for over the course of infection [4]. Similarly, Candida albicans hip mutants are selected in patients with oral thrush [9]. These findings indicate that persisters are largely responsible for failure to eradicate chronic infections [4].

Non-growing persisters make up a small part of the population: $10^{-6}$ to $10^{-4}$ in exponentially growing cultures and $\sim 10^{-2}$ in stationary phase [3,8]. Selection of Escherichia coli for mutants with increased persister formation produced a strain with two point mutations in hipA, G22S and D291A, (hipA7 allele) [10-14]. hipA is the toxin of the hipBA toxin/antitoxin (TA) pair. The antitoxin HipB represses the hipBA operon by cooperative binding to four operator sites $[10,11,15]$ and inactivates the toxin HipA. Ectopic expression of HipA causes multidrug tolerance [16]. Despite the strong phenotype of the gain-of-function allele hipA7 and HipA overexpression, a deletion of hipA did not produce a phenotype [12,16-19]. Similarly, ectopic expression of two other toxins, RelE and MazF, also strongly increased tolerance to antibiotics, whereas a deletion of the toxin gene had no phenotype [20]. This is not surprising given the considerable redundancy in these mRNA interferases - E. coli has at least 10 of them. Importantly, progressive deletion of all ten $\mathrm{mRNase}$ loci caused a pronounced decrease in the persister fraction [21].

One notable exception to the redundancy phenomenon is the toxin TisB, a membrane-acting peptide that causes dormancy by decreasing the pmf. Deletion of the type I TA module (small RNA antitoxin/protein toxin) tis $A B$ led to a pronounced decrease in the level of persisters. TisB is induced by the SOS response, and becomes the main mechanism of persister formation during SOS response, so a deletion has a phenotype $[22,23]$.

Unlike any other toxins of type II TA modules (protein antitoxin/protein toxin) which so far group mainly into either gyrase inhibitors (ParD, CcdB), mRNA interferases (RelE, MazF, YoeB, HicA, Doc) [24-26] or PIN domain fold proteins (VapC) [22,27], HipA is a kinase with a eukaryotic Ser/Thr kinase-like fold [16]. Replacing the conserved amino acids in the phosphorylation site $(\mathrm{S} 150 \mathrm{~A})$ or the $\mathrm{Mg}^{2+}$ - or catalytic binding sites (D332Q and D309Q respectively) abolishes the ability to confer growth arrest and antibiotic tolerance [16]. Elongation factor Tu (EF-Tu) was identified as a HipA target which points to a likely mechanism of HipA-mediated persister formation [15]. HipA phosphorylates EF-Tu, and $\mathrm{Thr}^{382}$-phosphorylated EF-Tu leads to stasis since it can no longer bind aminoacyl-tRNA [15]. Under the standard regime of batch culture growth the persistence function of HipA is 
masked by its tight interaction with HipB. To activate HipA, the antitoxin HipB has to be removed or degraded. Proteolytic regulation of the antitoxin has been demonstrated for several TA modules. In $E$. coli, the chromosomally encoded TA modules MazEF, RelBE, YefM/YoeB, HicAB, DinJ/YafQ and MqsRA are regulated by the AAA+ ATP-dependent proteases Lon and/or ClpPX [24,28-30]. ClpPA degrades the PhD antitoxin of the plasmid-encoded PhD/Doc TA module [22]. The other two ATPdependent proteases $\mathrm{HslVU}$ and $\mathrm{FtsH}$ do not have any known antitoxin substrates $[22,24]$. In this study we provide evidence that Lon is the main protease responsible for HipB degradation. Our data suggests that Lon recognizes the unstructured C-terminus of HipB.

\section{Results}

\section{Rapid degradation of HipB is dependent on the presence of Lon}

HipB does not share homology with any of the known antitoxins. Neutralization of its cognate toxin also differs mechanistically from other TA modules. Typically, the antitoxin contains an extended C-terminal stretch, which is structured only when in complex with its toxin [31-36]. Contacts usually involve residues near the active site of the toxin, which can simply be blocked by the antitoxin [33,35-37] or make it sterically impossible for the toxin to reach its target [31,32,34]. HipB, however, does not make any contacts with HipA near the active site. One HipB dimer binds two HipA molecules involving interactions with both the $\mathrm{N}$ and the $\mathrm{C}$ domain of HipA [15] (Fig. 1A). The $\mathrm{G}$ terminus of HipB is disordered (Fig. 1B) and remains unstructured in the presence of HipA [15]. To test whether proteolytic regulation is a shared characteristic of HipB with the typical antitoxins of the mRNA interferase and gyrase inhibitor TA modules, despite functional and structural differences, we measured the rate of in vivo degradation of HipB in wild type E. coli. Since endogenous HipB could not be detected by Western Blotting using a polyclonal antibody to HipB (data not shown), Nterminally six-his tagged $\mathrm{HipB}\left(\mathrm{His}_{6}-\mathrm{HipB}\right)$ was expressed from a plasmid containing an IPTG inducible promoter (pBRhipB). After $60 \mathrm{~min}$ of induction, protein synthesis was stopped by the addition of chloramphenicol and the rate of HipB proteolysis was determined by Western blotting (Fig. 2A). His 6 -HipB was degraded with a $t_{1 / 2}$ of $\approx 17 \mathrm{~min}$ in wild type cells confirming a rate of degradation characteristic for antitoxins [38,39]. Next, we transformed $\mathrm{pBR} h i p B$ into protease deficient strains lacking lon (KLE902); clpP (KLE903); or hslVU (KLE904) to identify a protease responsible for HipB degradation. We compared the rate of in vivo degradation of $\mathrm{HipB}$ in wild type to the rate of degradation in the protease deficient strains. Deletion of $\operatorname{clp} P$ or $h s l V U$ had a slight effect on HipB. The half life time of HipB was approximately $24 \mathrm{~min}$ in $\Delta c l p P$ and $28 \mathrm{~min}$ in $\Delta h s l V U$. Deletion of lon stabilized HipB (Fig. 2) ( $\mathrm{t}_{1 / 2}>200 \mathrm{~min}$ ), indicating that Lon is likely the main protease involved in HipB degradation in vivo. Since deletion of Lon protease had the strongest effect on the HipB turnover, we focused our studies on Lon dependent HipB degradation.

\section{HipB is a substrate of the ATP-dependent protease Lon}

To determine whether HipB is directly recognized and degraded by Lon we purified $\mathrm{His}_{6}$-Lon and $\mathrm{His}_{6}$-HipB for in vitro degradation studies. Lon degraded HipB with a $\mathrm{t}_{1 / 2}$ of $\approx 74 \mathrm{~min}$ in vitro confirming our findings obtained in vivo (Fig. 3A). The HipB decay however was much slower in the in vitro assay than in vivo. A difference between in vivo and in vitro degradation rate was also described for the antitoxin RelB which was degraded rapidly $\left(\mathrm{t}_{1 / 2} \approx 15 \mathrm{~min}\right)$ in a Lon dependent manner in vivo, whereas the in vitro half life time was $>60 \min [38,39]$. Substrate degradation by Lon requires ATP hydrolysis and $\mathrm{Mg}^{2+}$ [40]. HipB degradation was indeed dependent on the presence of ATP and $\mathrm{MgCl}_{2}$ in the reaction buffer demonstrating that degradation of $\mathrm{HipB}$ is specific to the addition of active enzyme to the buffer (Fig. 3B). The halflife of HipB in the in vitro assay without either $\mathrm{MgCl}_{2}$ or ATP was $250 \mathrm{~min}$ and $168 \mathrm{~min}$, respectively. There was some residual degradation in the absence of either ATP or $\mathrm{Mg} 2+$; however, both factors were clearly required for Lon-dependent degradation of HipB.

HipB functions as an inhibitor of HipA and as an autoregulator of the hipBA operon by cooperatively binding to the consensus

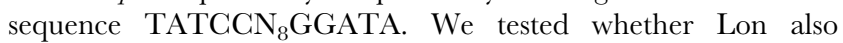
degrades HipB when it is bound to DNA or HipA. Addition of a 21 bp oligo ACTATCGCGTTAAGGGGATAG (sequence of top strand) spanning the hipBA operator sequence reduced the rate of degradation $\left(\mathrm{t}_{1 / 2}>200 \mathrm{~min}\right)$ ( $\mathrm{Fig}$. 3G), while addition of a control oligo (sequence of top strand ATGATGAGCTTTCAGAAGATC) showed little effect. Similarly, addition of purified $\mathrm{His}_{6}$-HipA slowed down the rate of HipB proteolysis $\left(t_{1}\right.$, ${ }_{2}>200 \mathrm{~min}$ ) as compared to a control protein (lysozyme) (Fig. 3D). Thus, HipB appears to be rapidly degraded only when it is free and not functioning as a transcriptional inhibitor of the hipBA operon or neutralizing the HipA protein.

\section{The unstructured C-terminus of HipB is critical for degradation}

The HipB dimerization interface is composed of a small hydrophobic core and the $\beta$-lid, a two-stranded intermolecular $\beta$ sheet that is followed by an unstructured 16 amino acid Cterminus (AKNASPESTEQQNLEW) [15]. Proteases typically bind disordered regions of their substrate, thus the unstructured $\mathrm{C}$ terminus appears to be an excellent recognition site for protease attack [15]. To test the hypothesis that the 16 residue G-terminal stretch is critical for degradation, we cloned a truncated HipB (HipB72) lacking the last 16 residues of HipB into pBR creating pBRhipB72. We measured the rate of in vivo degradation of HipB72 in wild type and $\Delta$ lon (KLE905 and KLE906, respectively) (Fig. 4). Interestingly, HipB72 is indeed substantially more stabile $\left(t_{1 / 2}>200 \mathrm{~min}\right)$ than full length HipB in wild type indicating that the unstructured $\mathrm{C}$ terminus of $\mathrm{HipB}$ is essential for degradation by Lon protease (Fig. 4A). As expected, full length HipB72 is also stable in $\Delta$ lon background. We purified the truncated HipB $\left(\mathrm{His}_{6}{ }^{-}\right.$ HipB72) and tested it in the Lon in vitro degradation assay. The effect was also noticeable though less pronounced in vitro. The halflife time of HipB changed from 74 min for full length HipB to $130 \mathrm{~min}$ in the mutant (Fig. 4B). To confirm that the unstructured $\mathrm{C}$ terminus of HipB is a degradation signal for Lon protease, we fused the $\mathrm{C}$ terminus of GFP with the unstructured C-terminal tail of HipB (creating pBRGFP-H, KLE908), and tested whether addition of the carboxy-terminal stretch of HipB (residues 73-88) causes degradation of GFP, which by itself is stable over the time period of the experiment $\left(\mathrm{t}_{1 / 2}>200 \mathrm{~min}\right)$ (Fig. 5). The GFP-HipB tail hybrid was much less stable with a half-life time of $\approx 53 \mathrm{~min}$ confirming that the $\mathrm{C}$-terminus of $\mathrm{HipB}$ is critical for rapid proteolysis of HipB.

\section{The C-terminus of HipB does not affect binding to either HipA or operator DNA}

The possibility that the last sixteen residues of HipB had additional functions beyond their role in protein stability was 

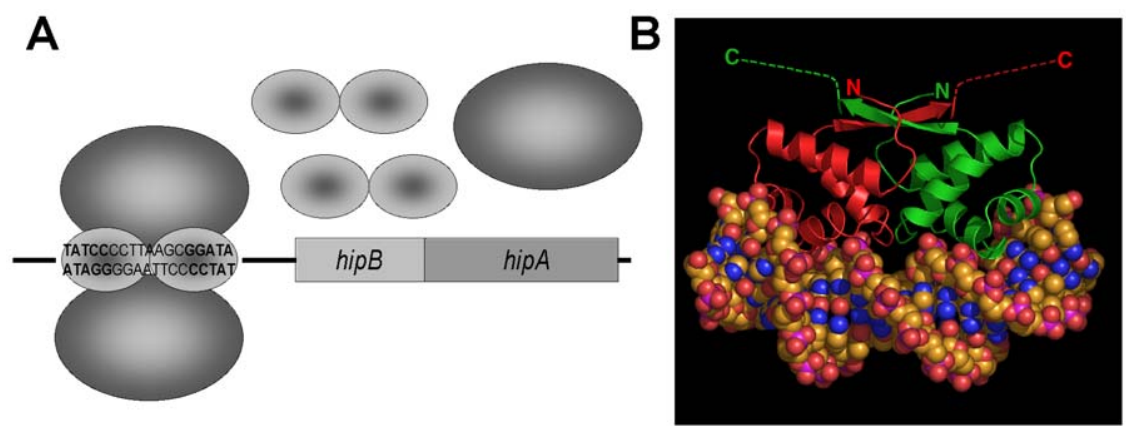

Figure 1. Overview of the hipBA locus of $E$. coli based on Schumacher et al. (A) Model of the hipBA operon. One of four operator sites is shown. (B) View of the crystal structure of the HipB dimer bound to a 21 base pair hipBA operator site (from reference [15]. One HipB subunit is colored green and the other red. The $\alpha$ helices are shown as coils and the $\alpha$ strands as arrows. The amino termini of each subunit are labelled $\mathrm{N}$ and the carboxy termini, C. The 16 C-terminal residues (73-88) are unstructured and residues $75-88$, which are disordered in the structure of the HipAHipB-DNA complex, are depicted as dashes and could easily extend from the body of HipB by more than $50 \AA$.

doi:10.1371/journal.pone.0039185.g001

considered especially in light of the finding that the C-terminal residue of HipB, W88, which is universally conserved (Fig. 6), interacted with a small surface pocket of HipA [41]. We first tested the effect of changing residue 88 to an alanine on HipB-DNA

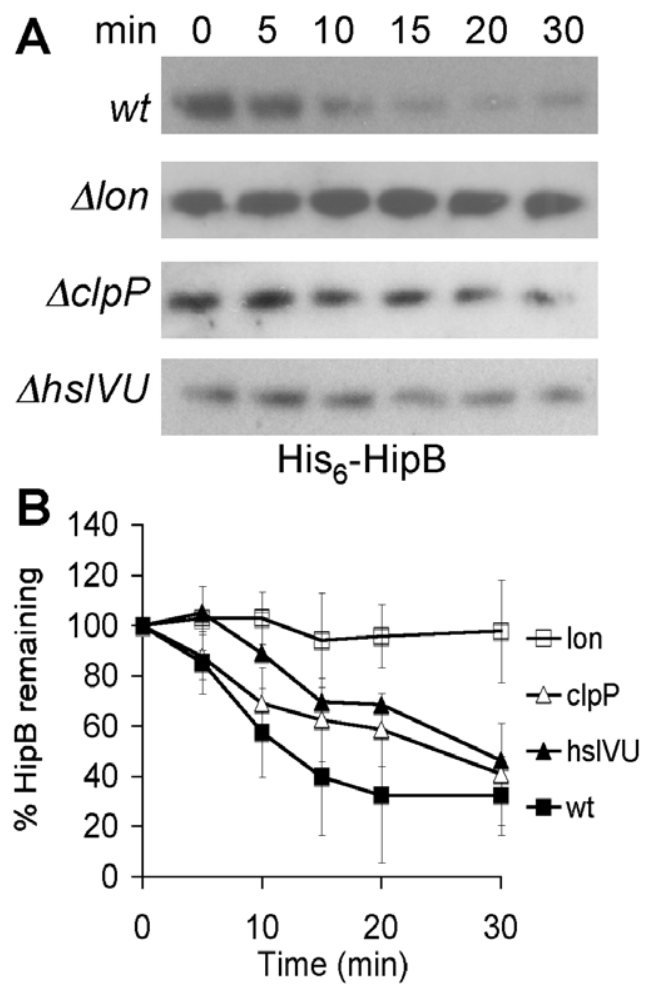

Figure 2. HipB proteolysis in $E$. coli wild type and protease deficient strains. HipB was expressed from pBRhipB in BW25113 (KLE901) and its lon::kan (KLE902), clpP::kan (KLE903) or hsIVU::FRT (KLE904) derivate. The strains were grown in LB medium, and at an $\mathrm{OD}_{600}$ of $0.31 \mathrm{mM}$ IPTG was added. After $1 \mathrm{~h}$ induction, protein synthesis was inhibited by the addition of $100 \mu \mathrm{g} / \mathrm{ml} \mathrm{Cam}$, and samples for Western blots were removed over the course of $30 \mathrm{~min}$. (A) The presence of HipB in whole cell lysates was detected with an anti-his antibody. (B) The rate of degradation was calculated from at least 3 independent experiments. Closed squares, KLE901 (wild type); open squares, KLE902 ( $\Delta$ lon); closed triangles KLE904 ( $\Delta h s / V U)$; open triangles, KLE903 $(\Delta c l p P)$.

doi:10.1371/journal.pone.0039185.g002 affinity. Using a fluorescence polarization-based assay and the hipBA $\mathrm{O}_{1} \mathrm{O}_{2}$ operator site, we determined that wild type HipB bound this sequence with a $\mathrm{K}_{\mathrm{d}}=0.6 \pm 0.1 \mathrm{nM}$ (Fig. 7A, 1). As anticipated from the HipA-HipB-DNA crystal structure [15], the HipB(W88A) protein bound this DNA with wild type HipB affinity $\left(\mathrm{K}_{\mathrm{d}}=0.9 \pm 0.4 \mathrm{nM}\right)$. Deletion of the last sixteen residues of HipB, (HipB72) also showed no change in DNA binding affinity $\left(\mathrm{K}_{\mathrm{d}}=0.4 \pm 0.1 \mathrm{nM}\right)$ (Fig. 7A, Table 1).

To test the hypothesis that W88 or other residues of the Cterminus contributes to HipA binding, we measured the dissociation constant of HipA for HipB (Fig. 7B and $\mathrm{C}$ ). The reporter molecule in this assay is the hipBA $O_{1} O_{2}$ sequence, which is saturated with HipB by using HipB concentrations 50 fold greater than $\mathrm{K}_{\mathrm{d}}$. HipA binding to HipB will result in a further, saturable increase in fluorescence polarization from which the HipA-HipB $\mathrm{K}_{\mathrm{d}}$ can be ascertained. The dissociation constant of HipA binding to HipB was $1 \mu \mathrm{M}$ under our experimental condition (Fig. 7B and G, Table 1). The $\mathrm{K}_{\mathrm{d}}$ of HipA for $\mathrm{HipB}(\mathrm{W} 88 \mathrm{~A})$ was identical to the wild type $\operatorname{HipB} \mathrm{K}_{\mathrm{d}}$ as was the $\mathrm{K}_{\mathrm{d}}$ of HipA for HipB72 (Fig. 7C, Table 1). Titration of HipA into $O_{1} O_{2}$ DNA in the absence of HipB results in linear, nonspecific DNA binding (data not shown). These results demonstrate that the C-terminus of HipB does not play a role in binding to either hipBA $O_{1} O_{2}$ DNA or HipA.

\section{Discussion}

The hipBA toxin/antitoxin locus shares several characteristics with other TA modules, such as the genetic organisation in an operon with the antitoxin overlapping the toxin by one base pair, tight regulation of the operon by the antitoxin and inhibition of the toxin by its antidote. In addition, ectopic expression of the toxin confers growth arrest, which can be overcome by antitoxin expression. However, the HipBA TA system does not group into the three common toxin families of RelBE-, the MazEF- and VapBC-like members. Toxin and antitoxin are structurally and mechanistically distinct from all other characterized TA pairs. HipA is a kinase, and HipB belongs to the Xre-helix-turn-helix family of transcriptional regulators. Binding of $\mathrm{HipA}-\mathrm{HipB}_{2}-\mathrm{HipA}$ to DNA introduces a $70^{\circ}$ bend in the operator [15]. In contrast to other antitoxins, HipB interacts with HipA via the $\mathrm{N}$ and $\mathrm{C}$ domain and the $\mathrm{G}$ terminus of HipB remains unstructured in the presence of the toxin [15]. Despite functional differences, regulation by proteolysis is a shared characteristic with all other protein-coding antitoxins. We find that HipB is a substrate of Lon protease since HipB is stabilized in the absence of Lon and 
A

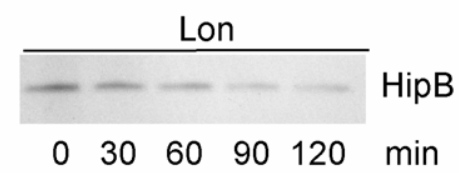

B

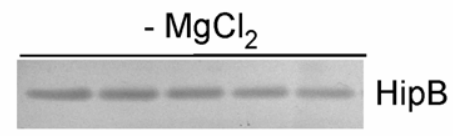

- ATP

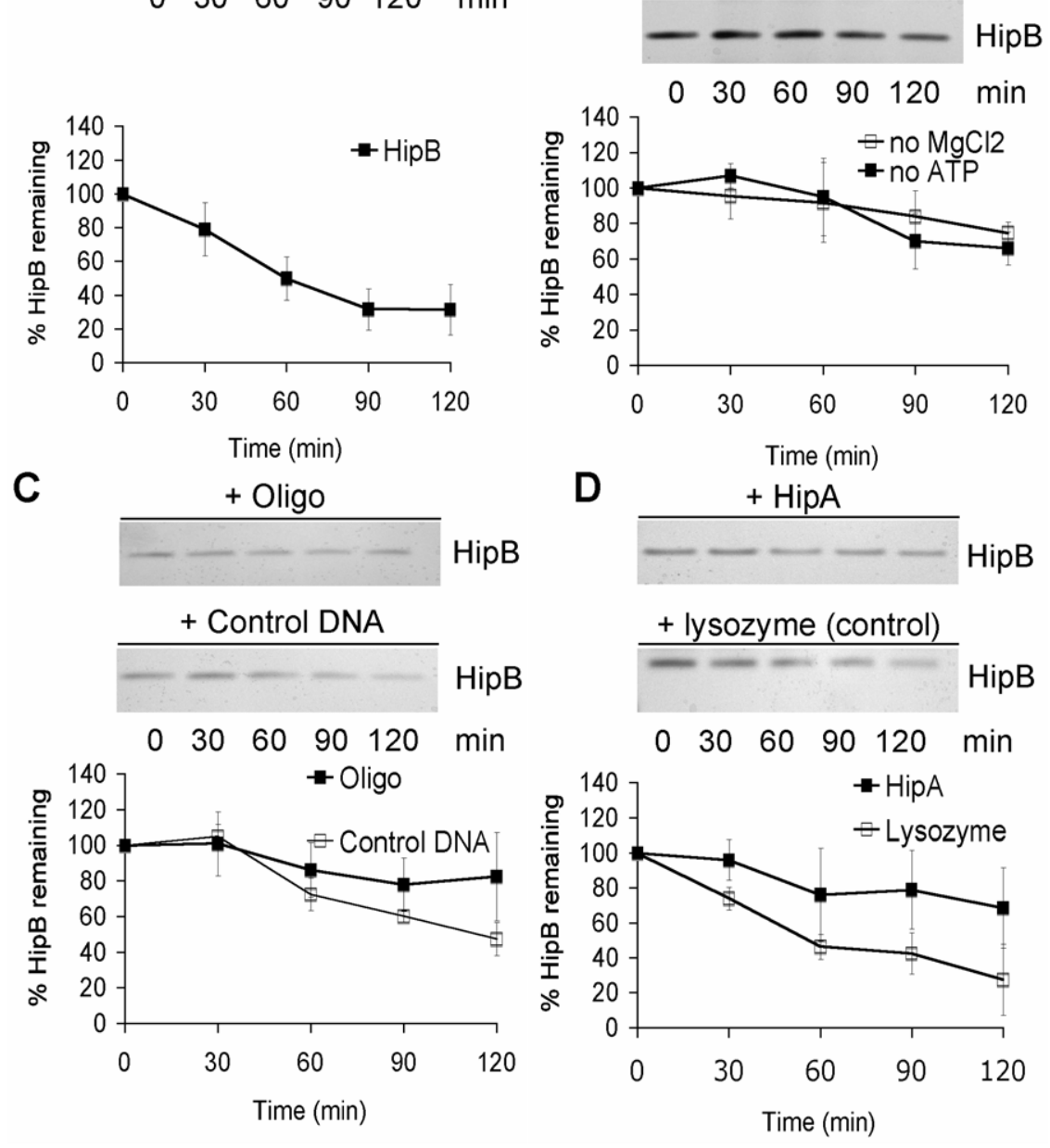

Figure 3. Lon degradation of HipB in vitro. $0.6 \mu \mathrm{M} \mathrm{His}{ }_{6}$-Lon and $0.48 \mu \mathrm{M} \mathrm{His}{ }_{6}-\mathrm{HipB}$ were incubated in reaction buffer at $37^{\circ} \mathrm{C}(50 \mathrm{mM}$ Tris- $\mathrm{HCl}$ (pH 8.0), $4 \mathrm{mM} \mathrm{ATP}, 7.5 \mathrm{mM} \mathrm{MgCl}$ ) for indicated times with or without the component specified and subjected to SDS-PAGE and silver staining

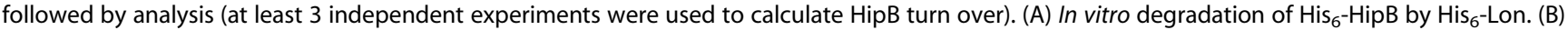
ATP or $\mathrm{MgCl}_{2}$ were omitted in the assay. Closed squares, no ATP; open squares no $\mathrm{MgCl}_{2}$. (C) Addition of an oligodeoxynucleotide encompassing the $21 \mathrm{bp}$ hip operator (closed squares) or control oligo (open squares) and (D) addition of His ${ }_{6} \mathrm{HipA}^{-}$(closed squares) or control protein (lysoszyme) (open squares) to the degradation assay.

doi:10.1371/journal.pone.0039185.g003

degraded by Lon in vitro. Under standard growth conditions HipB neutralizes HipA and represses transcription of the hipBA operon. However, when no new HipB is produced or Lon activity reaches elevated levels, HipB turnover results in free HipA. Shutdown of HipBA synthesis might be further regulated at the transcriptional or translational level, both diminishing the level of HipB decay and thus freeing up HipA. Currently, little is known about the activity of the hipBA promoter under different growth conditions. The position of an IHF binding site upstream of the hip operon suggests a level of transcriptional regulation beyond repression by HipB and HipBA binding to the operator. The activity of Lon protease is upregulated during stresses [42,43]. Polyphosphate (poly-P) binds to Lon and promotes degradation of ribosomal proteins (S2, L9, L13) while degradation of other proteins (e.g. SulA) is inhibited by poly-P $[44,45]$. Though a regulator of Lon activity that directs Lon to act on HipB has not been identified, it is possible that such a regulator exists. Increased activity of Lon will result in faster degradation of HipB faster and release free HipA. An additional possibility for regulating HipBA is that chaperones can potentially play a role in removing HipB from HipA. Though there is no direct evidence that a HipB is specifically regulated by chaperones, the persister level is 10 -fold reduced in a dnaK deletion [17]. If a chaperone sequesters $\mathrm{HipB}$, the persister level will be expected to be high due to free HipA. Subsequently, deletion of the chaperone will produce a low persister phenotype.

Irrespective of how it is released, free HipA will in turn phosphorylate EF-Tu and potentially act on additional targets leading to the shutdown of essential cellular functions and thus to dormancy. Phosphorylation of EF-Tu by HipA has been demonstrated only in vitro and remains to be confirmed in vivo. Additional targets of HipA are likely, and are currently a subject of investigation. 

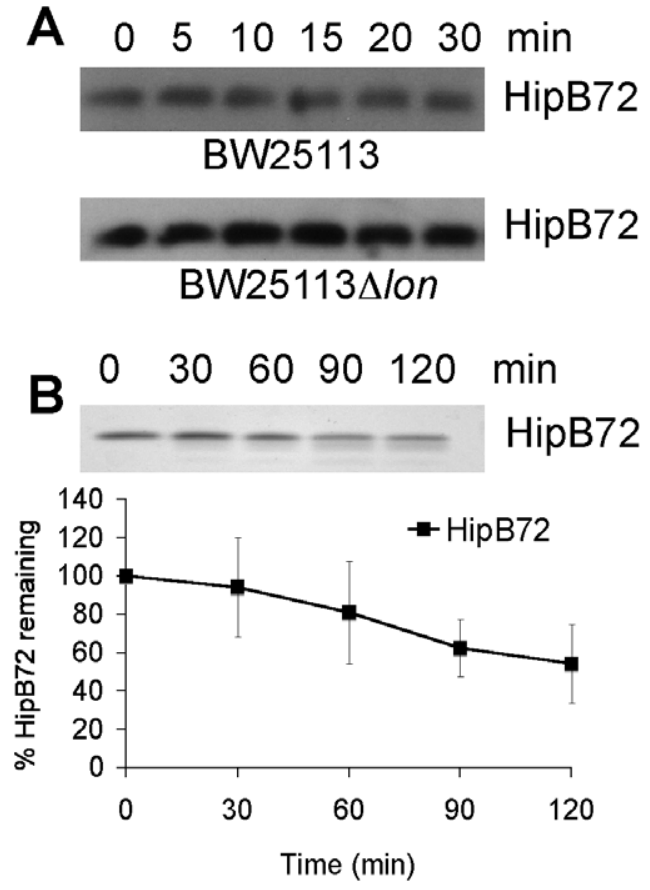

Figure 4. The $16 \mathrm{C}$-terminal amino acid residues of HipB are required for degradation. (A) Degradation of HipB72 in vivo. HipB72 was expressed from a pBRlacitac promoter in BW25113 (KLE905) and its lon::kan derivate (KLE906). Both strains were grown in LB medium, and at an $\mathrm{OD}_{600}$ of $0.31 \mathrm{mM}$ IPTG was added. After $1 \mathrm{~h}$ of induction, protein synthesis was inhibited by the addition of $100 \mu \mathrm{g} / \mathrm{ml} \mathrm{Cam}$, and samples for Western blots were removed over the course of $30 \mathrm{~min}$. (B) Degradation of HipB 72 in vitro. $\mathrm{His}_{6}-\mathrm{HipB} 72$ was purified and added to the Lon degradation assay. At least 3 independent experiments were performed to calculate HipB72 turnover. doi:10.1371/journal.pone.0039185.g004

In a recent study, Rotem et al., showed that if the amount of ectopically expressed HipA surpasses a certain threshold growth is arrested, whereas at low HipA levels growth is not affected, which leads to the formation of a distinct dormant; and growing subpopulation, correspondigly [46]. Considering our results, by controlling HipB degradation, Lon protease is the driving factor shifting HipA above or beyond the threshold levels.

Proteolytic degradation of antitoxins generally plays an important role in persister cell formation. Overexpression of Lon protease caused a 70 -fold increase in the level of persister cells compared to the wild type [21]. The increase in persistence dropped to a 4-fold difference in a strain lacking all ten mRNA endonuclease TA systems $(\Delta 10)$ in comparison to wild type control, indicating that Lon-mediated degradation of the antitoxins is responsible for the increase in the persister level [21]. Lon also regulates HipB degradation, and therefore HipBA likely contributes to the increase in persister formation under conditions when Lon is produced and HipB is degraded.

Another Lon substrate is the replication inhibitor CspD [47]. Interestingly, overexpression of CspD causes growth arrest [47] and therefore CspD might also be implicated in persistence. However, its role appears to be minor. The persister fraction in a strain overexpressing CspD increased less than 10-fold, and deletion of $\operatorname{csp} D$ caused a 2-fold change [48]. Given the known variability observed in the level of persisters, a robust persister phenotype of CspD remains to be established. While HipB degradation by Lon leads to HipA-mediated growth arrest, the situation is reversed for CspD. CspD-mediated growth arrest
$0102030405060 \mathrm{~min}$
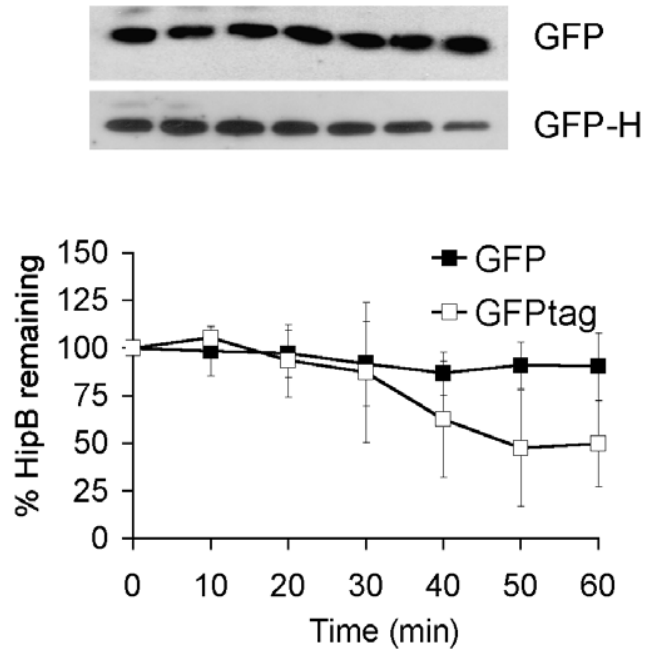

Figure 5. In vivo degradation of GFP and a GFP-HipB hybrid. GFP and GFP with C-terminal fusion to the $C$ terminus of HipB were expressed from a pBR/acitac promoter in BW25113 (KLE907 and KLE908, respectively). The strains were grown in $\mathrm{LB}$ medium, and at an $\mathrm{OD}_{600}$ of $0.31 \mathrm{mM}$ IPTG was added. After $1 \mathrm{~h}$ of induction, protein synthesis was inhibited by the addition of $100 \mu \mathrm{g} / \mathrm{ml} \mathrm{Cam}$, and samples for Western blots were removed over the course of $60 \mathrm{~min}$. Closed squares, GFP; open squares GFP-H (GFP-HipB(73-88)). The graph represents the average of five independent experiments.

doi:10.1371/journal.pone.0039185.g005

reversed by Lon, suggesting a possible resuscitation mechanism. It remains to be established how Lon itself is regulated to control toxin degradation.

Our data show that a disordered C-terminus of HipB serves as a degradation signal for the Lon protease. We must note that these results were obtained with an N-terminal his-tagged HipB. It is unlikely however that this N-terminal his-tag affected degradation. The common cause of artifacts in determining degradation of ectopically expressed (including tagged) proteins stems from formation of aggregates, which include the overexpressed protein, chaperones and proteases. This is the case for a number of membrane-associated proteins, whereas overexpression of soluble GST-GFP did not result in aggregation [49]. $\mathrm{His}_{6}$-HipB is soluble and we have no indication that aggregates are formed and are interfering with degradation of HipB. HipB and CspD both have in common an extended or disordered C-terminus [47]. While Langklotz and Narberhaus do not observe any differences in stability of CspD point mutations of non-polar residues in the $\mathrm{C}$ terminal region (V73D-A74D) and truncations of the $\mathrm{G}$ terminus (lacking the last five, seven, and eleven residues, respectively), we find that a HipB mutant lacking the conformationally flexible $\mathrm{C}$ terminus is stabilized suggesting that the $\mathrm{C}$-terminus is a signal sequence for Lon pointing to a different mechanisms of substrate recognition for these two proteins. The possibility that the HipB C-terminus functions beyond acting as a degradation tag has been raised by Evdokimov and colleagues [41]. In the structures of the HipB-HipA and HipA-HipB-DNA complexes [15], HipB residue W88 was found bound in a pocket on the HipA surface approximately $14 \AA$ from the catalytic pocket. This led Evdokimov to suggest that this residue might be important for HipA-HipB interaction as well as potentially acting as an allosteric regulator of HipA activity [41]. Our binding assays show that residue W88 and indeed residues 73 through 88 play no role in HipA binding to HipB as the wild type HipB, HipB(W88A) and HipB72 have 

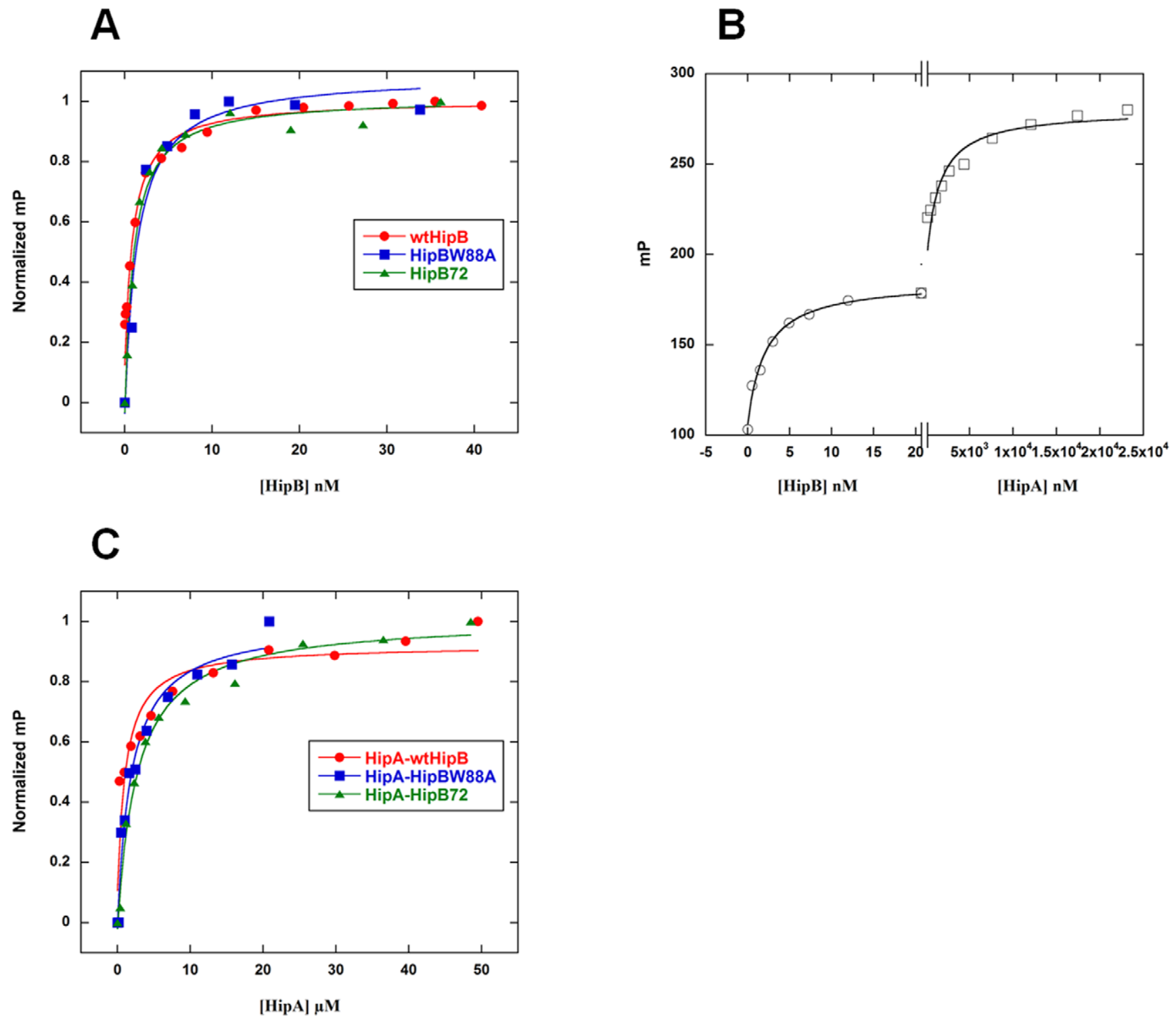

Figure 6. Multiple sequence alignment of selected HipB proteins from a variety of Gram-negative bacteria. CLUSTALW and CLC Main Workbench were used for the alignment and graphic representation, respectively. HipB sequences were downloaded from NCBI database. doi:10.1371/journal.pone.0039185.g006

identical binding affinities for HipA. Such similar binding affinities would also seem to eliminate the possibility that HipB residue W88 regulates HipA allosterically. However, HipA binding to HipB does appear to block access of Lon to HipB (Fig. 3D). This is not surprising as the $(\mathrm{HipA})_{2}$-HipB dimer structure shows that the $\mathrm{C}$ terminus of HipB is partially shielded from the solvent, which would hinder its access by Lon [15,41]. Thus, we can conclude that the last sixteen residues of HipB serve as a highly efficient tag for the destruction of the antitoxin HipB by Lon protease but only after its dissociation from the HipA toxin and the hipBA operator. This requirement for two dissociation events before HipB degradation might serve to ensure that the switch to persistence is not thrown accidently.

\section{Materials and Methods}

\section{Bacterial strains and growth conditions}

The bacterial strains and plasmids used in this study and their relevant characteristics are listed in Table 2. Strains were grown in LB medium unless otherwise noted. When required, LB broth or LB agar were supplemented with ampicillin (100 $\mu \mathrm{g} / \mathrm{ml}$, Amp) and chloramphenicol $(30 \mu \mathrm{g} / \mathrm{ml}$, Cam).

\section{Plasmid constructions}

Primers PlhishipBXbaI (CGGTCTAGATAAGGAGATATATGG AATAATGGACGACGACGACGACGACATGAGCTTTCAGAAGATCTA) and P2hipBEcoRI (CGGGAATTCTTACGACTCGAGATTTTGCTG) or P2hipBsEcoRI (CGGG AATTCGTGGCATAGGGTCATTGAGAG) were used to amplify hipB from E. coli MG1655; P1GFPXbaI (CGGTCTAGATAAGGAGATATATGGAATAATGAG-

TAAAGGAGAAGAACT) and P2GFPEcoRI (CGGGAATTCT- 


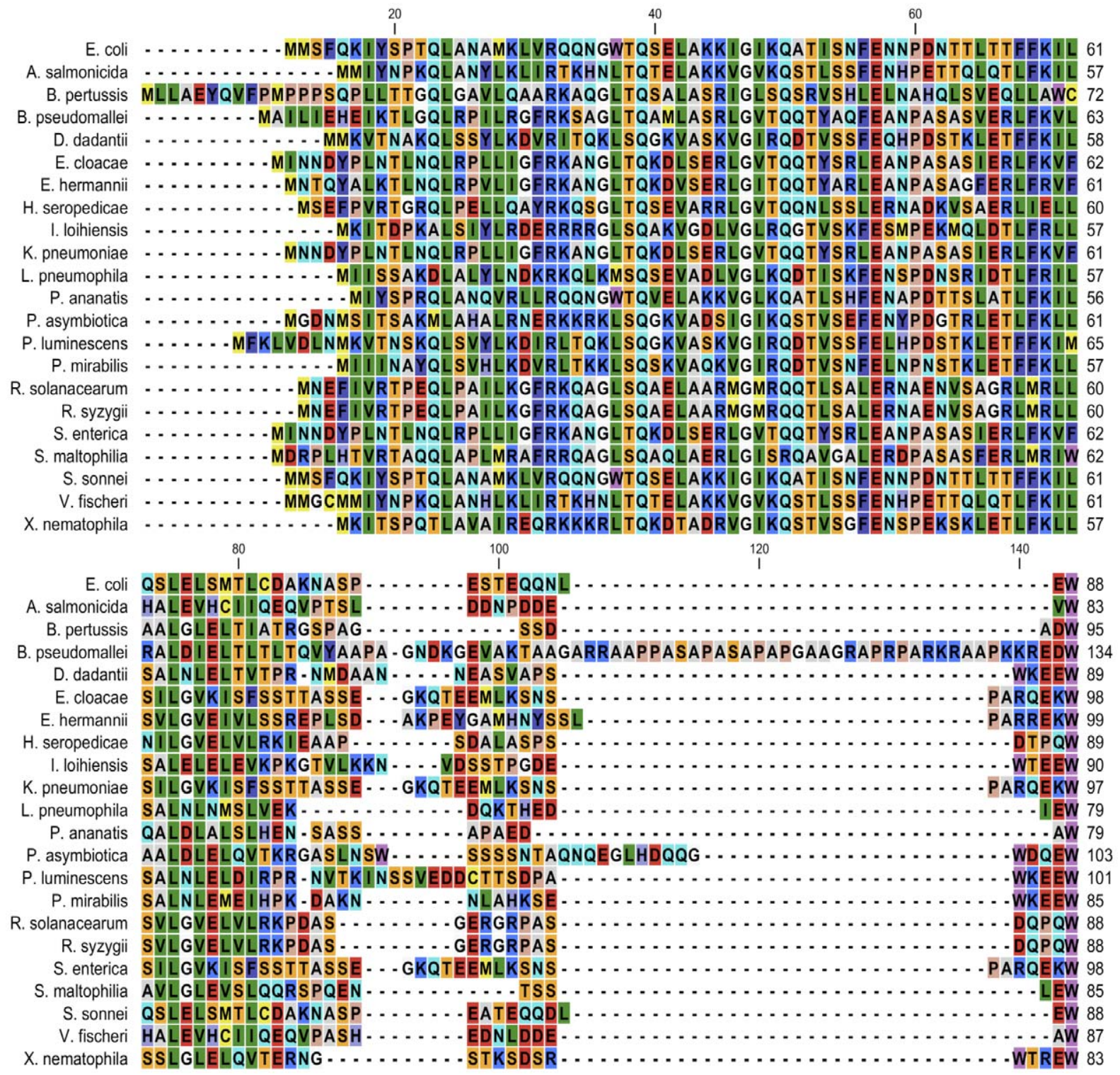

Figure 7. HipB, HipB(W88A) and HipB72 bind hipBA operator DNA or HipA identically. (A) Wild type HipB protein (red closed circle), $\mathrm{HipB}(\mathrm{W} 88 \mathrm{~A})$ protein (blue closed square) or the HipB72 C-terminal truncation protein (green closed triangle) was titrated into fluoresceinated hipBA $\mathrm{O}_{1} \mathrm{O}_{2}$ operator sequence and the change in fluorescence polarization (normalized millipolarization, $\mathrm{mP}$ ) plotted as a function of the concentration of the titrant. The typical change in $\mathrm{mP}$ of each titration was between 60 and 80 units. The correlation coefficients for each curve fitting were $0.98,0.99$ and 0.99 , respectively. (B) Wild type HipA protein was titrated into hipBA 0102 DNA after the DNA was prebound by $20 \mathrm{nM}$ wild type HipB monomer. Note that the concentration range is different in the left half and right half of the binding isotherms, with HipB titrations in the nanomolar range and HipA titrations in the micromolar range. (C) Wild type HipA protein was titrated into solutions containing $1 \mathrm{nM}$ fluorescently labelled hipBA $O_{1} \mathrm{O}_{2}$ DNA

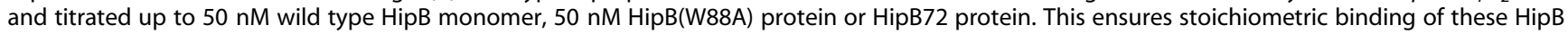
proteins to the DNA. Thus, the resulting binding affinity is formally between HipA and HipB that is bound specifically to hipBA DNA. The change in mP of each titration was between 88 and 150 units. The correlation coefficients for each curve fitting were $0.95,0.99$ and 0.99 , respectively. A representative binding isotherm is shown for each protein binding to DNA or to the HipB-hipBA $O_{1} O_{2}$ complex. doi:10.1371/journal.pone.0039185.g007

\begin{abstract}
TACGACTCGAGATTTTGCTGTTCTGTT GATTCTGGCGAGGCATTTTTCGCTTTGTATAGTTCATCGATGG) or P2GHEcoRI (CG GGAATTCTTATTTGTATAGTTCATCCATGC) were used to amplify GFP from pUA66. The PCR products were digested with $X b a \mathrm{I}$ and $E c o$ RI and ligated into $X b a \mathrm{I}$ and $E_{c o R I}$ sites of pBRlac $\mathrm{tac}$ creating $\mathrm{pBR} h i p B$ or $\mathrm{pBR} h i p B 72$ and pBRGFP or pBRGFP-H, respectively.
\end{abstract}

\section{Mutant generation}

The HipB(W88A) mutant and the truncation mutant HipB72 (deletion of all amino acid residues after position 72) were generated using site directed mutagenesis. Primers used for the mutagenesis are as follows:

5' - AACGTGGAAGCGTAACTGGAGGATCGG and

5'- CGGATCGTGGAGTTACGGTTCGAGGTT for HipB(W88),

5'-GGACGGCATATGAGTTTTCAGAAAATCTA-

TAGTCG and 
Table 1. Dissociation constants of HipB-DNA and HipA-(HipBDNA)*.

\begin{tabular}{lll}
\hline & & \\
\hline & $0_{1} O_{2}$ DNA $(\mathbf{n M})$ & HipA $(\mu \mathbf{M})$ \\
\hline HipB WT & $0.6 \pm 0.1$ & $1.0 \pm 0.6$ \\
HipB(W88A) & $0.9 \pm 0.4$ & $1.2 \pm 0.3$ \\
HipB72 & $0.4 \pm 0.1$ & \pm 0.1 \\
\hline
\end{tabular}

*The binding constants are the average of at least three separate measurements.

doi:10.1371/journal.pone.0039185.t001
5'-GAACGGCTCGAGTTAATCACACAGCGTCATCGAC for HipB72). The generation of mutants was verified by DNA sequencing.

\section{Strain construction}

Precise deletion-replacement of hslVU was created by the method of Datsenko and Wanner [50].

\section{Protein expression and purification}

N-terminally hexa-histidine $\left(\right.$ his $_{6}$ )-tagged HipB, HipB72, HipA and Lon were purified using strains and plasmids indicated in Table 2. Strains were induced by addition of $0.2 \%$ arabinose or $1 \mathrm{mM}$ IPTG for $4 \mathrm{~h}$. Cells were lysed by sonication in the presence of $1 \mathrm{mg} \bullet \mathrm{ml}^{-1}$ lysozyme. Protein extracts were applied to a Ni-NTA resin (QIAGEN). The columns were washed with

Table 2. Strains and plasmids used in this study.

\begin{tabular}{|c|c|c|}
\hline Strain or plasmid & Relevant characteristics & Source or reference \\
\hline \multicolumn{3}{|l|}{ E. coli K-12 strains } \\
\hline MG1655 & $\mathrm{F}^{-} \lambda^{-} i l v G r f b-50 r p h-1$ & [53] \\
\hline BW25113 & lacla rrnB3 lacZ4787 hsdR514 $\Delta($ araBAD) $567 \Delta($ rhaBAD)568 rph-1 & [54] \\
\hline BW25113 Alon & $\Delta / o n: k a n$ & Keio collection, [55] \\
\hline $\mathrm{BW} 25113 \Delta c / p P$ & $\Delta c l p P:: k a n$ & Keio collection, [55] \\
\hline BW25113 $\Delta h s / V U$ & $\Delta h s I V U:: F R T$ & This study \\
\hline KLE901 & BW25113 pBRhipB & This study \\
\hline KLE902 & BW25113 $\Delta$ lon pBRhipB & This study \\
\hline KLE903 & BW25113 $\Delta c l p P:: k a n$ pBRhipB & This study \\
\hline KLE904 & BW25113 $\Delta h s / V U:: F R T$ pBRhipB & This study \\
\hline KLE905 & BW25113 pBRhipB72 & This study \\
\hline KLE906 & BW25113 $\Delta$ lon pBRhipB72 & This study \\
\hline KLE907 & BW25113 pBRGFP & This study \\
\hline KLE908 & BW25113 pBRGFP-H & This study \\
\hline KLE319 & MG1655 $\Delta$ hipA pBADhipA & [16] \\
\hline AG1 pCA24N/on & $\begin{array}{l}\left.\text { AG1 (endA1 recA1 gyrA96 thi-1 relA1 glnV44 hsdR17(r } r_{K}^{-} m_{K}^{+}\right) \\
\text {pCA24N/on, GFP free }\end{array}$ & ASKA library, [56] \\
\hline KLE909 & BL21(DE3)pLysS pEThipA & [41] \\
\hline KLE910 & BL21(DE3)pLysS pEThipB & [15] \\
\hline KLE911 & BL21(DE3)pLysS pEThipB(W88A) & This study \\
\hline KLE912 & BL21(DE3)pLysS pEThipB72 & This study \\
\hline \multicolumn{3}{|l|}{ Plasmids } \\
\hline pBR/acitac & bla lacl ${ }^{9}$ Ptac pBR322 ori & [57] \\
\hline pBAD33 & cat araC $P_{\mathrm{BAD}} \mathrm{PACYC} 184$ ori & [58] \\
\hline pUA66 & pSC101 ori, GFP reporter plasmid carrying GFPmut2, $\mathrm{Km}^{\mathrm{R}}$ & [59] \\
\hline pCA24N/on & pCA24N/on, GFP free, $\mathrm{Cm}^{\mathrm{R}}$ & ASKA library, [56] \\
\hline pBRhipB & pBRlacitac-hipB & This study \\
\hline pBRhipB72 & pBRlacitac-hipB72 & This study \\
\hline pBRGFP & pBRlacitac-GFP & This study \\
\hline pBRGFP-H & pBRlacitac-GFPhipB(73-88) & This study \\
\hline pBADhipA & pBAD33-hipA & [16] \\
\hline pEThipA & pET28a-hipA & [41] \\
\hline pEThipB & pET15b-hipB & [15] \\
\hline pEThipB(W88A) & pET15b-hipB w88a & This study \\
\hline pEThipB72 & pET15b-hipB72 & This study \\
\hline
\end{tabular}


buffer containing $20 \mathrm{mM}$ imidazole, and eluted with buffer containing $250 \mathrm{mM}$ imidazole. The eluted protein was concentrated and dialyzed with protein storage buffer $(50 \mathrm{mM}$ Tris- $\mathrm{HCl}$, pH 8, $250 \mathrm{mM} \mathrm{NaCl}, 5 \mathrm{mM}$ dithiothreitol [DTT], 5\% glycerol) [51].

Proteins used for fluorescence polarization-based binding assays were expressed and purified as described below. Plasmid DNA (pET28a-HipA or pET15b-HipB) was transformed into BL21(DE3)pLysS competent cells, which were plated onto LB agar containing $50 \mu \mathrm{g} \bullet \mathrm{mL}^{-1}$ kanamycin for HipA expressing cells and $100 \mu \mathrm{g} \bullet \mathrm{mL}^{1}$ ampicillin for HipB expressing cells, respectively. All cells were allowed to grow at $37^{\circ} \mathrm{C}$ with appropriate antibiotics. A single colony was selected from each plate and inoculated into $250 \mathrm{~mL} \mathrm{LB}$ for overnight growth. $40 \mathrm{~mL}$ cells were transferred into 1.5 L LB and were incubated until the $\mathrm{OD}_{600}$ reached 0.6. Proteins were induced for four hours by adding $1 \mathrm{mM}$ IPTG. Cells were harvested by centrifugation at $4,000 \times \mathrm{g}, 4^{\circ} \mathrm{C}$ for 10 minutes and the harvested cells were stored in $-80^{\circ} \mathrm{C}$. The frozen cells were thawed and resuspended in $50 \mathrm{~mL}$ buffer A (20 mM Tris-HCl pH 7.5, $300 \mathrm{mM} \mathrm{NaCl}, 5 \%$ glycerol) containing one protease inhibitor tablet and $20 \mu \mathrm{g} \cdot \mathrm{mL}^{-1}$ DNase I (Roche). After homogenizing the cells with a dounce tissue grinder, cells were disrupted using a microfluidizer (model M110L, Microfluidics) and centrifuged at $31,000 \times \mathrm{g}, 4^{\circ} \mathrm{C}$ for 1 hour. The supernatant was applied to $3 \mathrm{~mL}$ Ni-NTA affinity resin equilibrated with Buffer A and allowed to run by gravity. The column was washed overnight with buffer A containing $20 \mathrm{mM}$ imidazole at $0.5 \mathrm{~mL} \cdot \mathrm{min}^{-1}$ flow rate. Proteins were eluted with 50-200 mM imidazole gradient. Protein homogeneity was estimated by a SDS-PAGE gel with coomassie staining. Buffer exchange and protein concentration were achieved using Amicon ultra centrifugal filter (Millipore). Protein concentrations were determined by Bradford using BSA as the standard.

\section{Fluorescence polarization-based binding affinity assays}

Fluorescence polarization experiments were performed to determine the binding affinities of $\mathrm{HipB}$, the HipB(W88A) mutant and HipB72 proteins for DNA and HipA [52]. A DNA oligodeoxynucleotide containing two hipB operator sites $\left(O_{1} O_{2}\right)$ with the sequence

(5'-TTATCGGCTTAAGGGGATATTATAAGTTT-

TATCGTTTAGTGAGGATAA- $3^{\prime}$ ) and labeled with 6-carboxyfluorescein at $5^{\prime}$-end was purchased (Integrated DNA Technologies Inc) and used without further purification. The labeled DNA was heated to $95^{\circ} \mathrm{C}$ for two to five minutes in the presence of an equal amount of the unlabelled complementary strand. The strands were annealed by cooling slowly at room temperature. DNA binding was carried out in $0.5 \mathrm{~mL}$ binding buffer A $(20 \mathrm{mM}$ Tris-HCl pH 7.5, $100 \mathrm{mM} \mathrm{NaCl}$ and 5\% glycerol) in which $1.0 \mathrm{nM}$ labeled DNA was included. Changes in fluorescence polarization were monitored using a Panvera Beacon 2000 (Panvera Corporation) as a function of increasing concentration

\section{References}

1. Balaban NQ Merrin J, Chait R, Kowalik L, Leibler S (2004) Bacterial persistence as a phenotypic switch. Science 305: 1622-1625.

2. Bigger JW (1944) Treatment of staphylococcal infections with penicillin. Lancet ii: $497-500$.

3. Lewis K (2007) Persister cells, dormancy and infectious disease. Nat Rev Microbiol 5: 48-56.

4. Lewis K (2010) Persister Cells. Annu Rev Microbiol.

5. Keren I, Kaldalu N, Spoering A, Wang Y, Lewis K (2004) Persister cells and tolerance to antimicrobials. FEMS Microbiol Lett 230: 13-18.

6. Wiuff C, Zappala RM, Regoes RR, Garner KN, Baquero F, et al. (2005) Phenotypic tolerance: antibiotic enrichment of noninherited resistance in bacterial populations. Antimicrob Agents Chemother 49: 1483-1494. of HipB. Binding was done at $10^{\circ} \mathrm{C}$. The binding affinity of HipA for HipB was determined by titrating HipA into a solution of $50 \mathrm{nM}$ HipB, which was pre-incubated with $1 \mathrm{nM}$ labeled DNA for 2 minutes, in $0.5 \mathrm{~mL}$ binding buffer $\mathrm{A}$. The concentration of $\mathrm{HipB}$ assured the stoichiometric binding of the $O_{1} O_{2}$ DNA site, as $50 \mathrm{nM}$ HipB is nearly 100 fold more than the $\mathrm{K}_{\mathrm{d}}$ that was determined for HipB- $O_{1} O_{2}$ binding. The wavelengths used for excitation and emission were $490 \mathrm{~nm}$ and $530 \mathrm{~nm}$, respectively. Dissociation constants were obtained as described previously [52]. Briefly, the increase in fluorescence millipolarization $\left(\mathrm{mP}=\mathrm{P} \cdot 10^{-3}\right.$, where $\mathrm{P}$ is polarization) as a function of the increasing HipB or HipA concentration is fit using the curve fitting function of Kaleidagraph and the equation: $\mathrm{P}=\left\{\left(\mathrm{P}_{\text {bound }}-\mathrm{P}_{\text {free }}\right)\right.$ $[$ HipB-DNA $] /\left(\mathrm{K}_{\mathrm{d}}+[\right.$ HipB-DNA] $\left.)\right\}+\mathrm{P}_{\text {free }}$, where $\mathrm{P}$ is the polarization measured at a given concentration of $\mathrm{HipB}, \mathrm{P}_{\text {free }}$ is the initial polarization of the free DNA, and $\mathrm{P}_{\text {bound }}$ is the maximum polarization of specifically bound DNA. For determination of the HipA affinity for HipB, the same equation is used but the HipBDNA term is replaced by HipA-(HipB-DNA). Nonlinear least squares analysis is used to determine $\mathrm{P}_{\text {bound }}$ and $\mathrm{K}_{\mathrm{d}}$.

\section{In vivo degradation and Western Blot analysis}

The rate of degradation of HipB, HipB72, GFP and GFP-H was determined using samples from exponentially growing cells. Expression of proteins from pBRlacitac was induced by addition of $1 \mathrm{mM}$ IPTG at $\mathrm{OD}_{600}$ of 0.3 . After $1 \mathrm{~h}$ induction, protein synthesis was inhibited by the addition of $100 \mu \mathrm{g} / \mathrm{ml} \mathrm{Cam}$, and samples were removed at indicated time points. Same sample volume was loaded in each well of an SDS-PAGE gel. Protein levels were detected by Western blotting using either a monoclonal His-tag antibody (EMD Biosciences) or polyclonal antibody to GFP (Abcam) and a polyclonal goat-anti mouse IgG AP conjugate.

\section{In vitro degradation assay}

To monitor degradation of HipB, $\mathrm{His}_{6}$-Lon $(0.6 \mu \mathrm{M})$ and $\mathrm{His}_{6}{ }^{-}$ HipB $(0.48 \mu \mathrm{M})$ or $\mathrm{His}_{6}$-HipB72 $(0.48 \mu \mathrm{M})$ were added to a degradation buffer [50 mM Tris-HCl (pH 8.0), $4 \mathrm{mM}$ ATP, $7.5 \mathrm{mM} \mathrm{MgCl} 2]$ and incubated at $37{ }^{\circ} \mathrm{C}$. When indicated, $\mathrm{His}_{6}{ }^{-}$ HipB was mixed with either $\mathrm{His}_{6}-\mathrm{HipA}(0.48 \mu \mathrm{M})$ lysozyme $(0.48 \mu \mathrm{M})$ or duplex DNA containing a $21 \mathrm{bp}$ hipB operator site $(0.24 \mu \mathrm{M})$ (sequence of the top strand ACTATCGCGTTAAGGGGATAG) or a control deoxynucleotide (sequence of the top strand ATGATGAGGTTTGAGAAGATG). Samples were removed at indicated times, and analyzed by SDS-PAGE. The same sample volume was loaded in each well.

\section{Author Contributions}

Conceived and designed the experiments: SH KL MAS RGB. Performed the experiments: SH TJY JM. Analyzed the data: SH MV KL MAS RGB TJY JM. Contributed reagents/materials/analysis tools: SH TJY JM RGB. Wrote the paper: SH MV KL TJY MAS RGB KL.

7. Brooun A, Liu S, Lewis K (2000) A dose-response study of antibiotic resistance in Pseudomonas aeruginosa biofilms. Antimicrob Agents Chemother 44: 640-646.

8. Spoering AL, Lewis K (2001) Biofilms and planktonic cells of Pseudomonas aeruginosa have similar resistance to killing by antimicrobials. J Bacteriol 183: 6746-6751.

9. LaFleur MD, Qi Q, Lewis K (2010) Patients with long-term oral carriage harbor high-persister mutants of Candida albicans. Antimicrob Agents Chemother 54: 3944

10. Black DS, Irwin B, Moyed HS (1994) Autoregulation of hip, an operon that affects lethality due to inhibition of peptidoglycan or DNA synthesis. J Bacteriol 176: 4081-4091 
11. Black DS, Kelly AJ, Mardis MJ, Moyed HS (1991) Structure and organization of hip, an operon that affects lethality due to inhibition of peptidoglycan or DNA synthesis. J Bacteriol 173: 5732-5739.

12. Moyed HS, Bertrand KP (1983) hipA, a newly recognized gene of Escherichia coli K-12 that affects frequency of persistence after inhibition of murein synthesis. J Bacteriol 155: 768-775.

13. Moyed HS, Broderick SH (1986) Molecular cloning and expression of hipA, a gene of Escherichia coli K-12 that affects frequency of persistence after inhibition of murein synthesis. J Bacteriol 166: 399-403.

14. Scherrer R, Moyed HS (1988) Conditional impairment of cell division and altered lethality in hipA mutants of Escherichia coli K-12. J Bacteriol 170: 3321 3326 .

15. Schumacher MA, Piro KM, Xu W, Hansen S, Lewis K, et al. (2009) Molecular mechanisms of HipA-mediated multidrug tolerance and its neutralization by HipB. Science 323: 396-401.

16. Correia FF, D’Onofrio A, Rejtar T, Li L, Karger BL, et al. (2006) Kinase activity of overexpressed HipA is required for growth arrest and multidrug tolerance in Escherichia coli. J Bacteriol 188: 8360-8367.

17. Hansen S, Lewis K, Vulić M (2008) The role of global regulators and nucleotide metabolism in antibiotic tolerance in Escherichia coli. Antimicrob Agents Chemother.

18. Korch SB, Henderson TA, Hill TM (2003) Characterization of the hipA7 allele of Escherichia coli and evidence that high persistence is governed by (p)ppGpp synthesis. Mol Microbiol 50: 1199-1213.

19. Korch SB, Hill TM (2006) Ectopic overexpression of wild-type and mutant hipA genes in Escherichia coli: effects on macromolecular synthesis and persister formation. J Bacteriol 188: 3826-3836.

20. Keren I, Shah D, Spoering A, Kaldalu N, Lewis K (2004) Specialized persister cells and the mechanism of multidrug tolerance in Escherichia coli. J Bacteriol 186: 8172-8180.

21. Maisonneuve E, Shakespeare LJ, Jorgensen MG, Gerdes K (2011) Bacterial persistence by RNA endonucleases. Proc Natl Acad Sci U S A.

22. Buts L, Lah J, Dao-Thi MH, Wyns L, Loris R (2005) Toxin-antitoxin modules as bacterial metabolic stress managers. Trends Biochem Sci 30: 672-679.

23. Dorr T, Vulic M, Lewis K (2010) Ciprofloxacin causes persister formation by inducing the TisB toxin in Escherichia coli. Plos Biol 8: e1000317.

24. Gerdes K, Christensen SK, Lobner-Olesen A (2005) Prokaryotic toxin-antitoxin stress response loci. Nat Rev Microbiol 3: 371-382.

25. Jorgensen MG, Pandey DP, Jaskolska M, Gerdes K (2009) HicA of Escherichia coli defines a novel family of translation-independent mRNA interferases in bacteria and archaea. J Bacteriol 191: 1191-1199.

26. Winther KS, Gerdes K (2009) Ectopic production of VapCs from Enterobacteria inhibits translation and trans-activates YoeB mRNA interferase. Mol Microbiol 72: 918-930.

27. Arcus VL, Rainey PB, Turner SJ (2005) The PIN-domain toxin-antitoxin array in mycobacteria. Trends Microbiol 13: 360-365.

28. Christensen SK, Pedersen K, Hansen FG, Gerdes K (2003) Toxin-antitoxin loci as stress-response-elements: ChpAK/MazF and ChpBK cleave translated RNAs and are counteracted by tmRNA. J Mol Biol 332: 809-819.

29. Prysak MH, Mozdzierz CJ, Cook AM, Zhu L, Zhang Y, et al. (2009) Bacterial toxin YafQ is an endoribonuclease that associates with the ribosome and blocks translation elongation through sequence-specific and frame-dependent mRNA cleavage. Mol Microbiol 71: 1071-1087.

30. Wang X, Kim Y, Hong SH, Ma Q, Brown BL, et al. (2011) Antitoxin MqsA helps mediate the bacterial general stress response. Nat Chem Biol 7: 359-366.

31. Cherny I, Overgaard M, Borch J, Bram Y, Gerdes K, et al. (2007) Structural and thermodynamic characterization of the Escherichia coli RelBE toxinantitoxin system: indication for a functional role of differential stability. Biochemistry 46: 12152-12163.

32. Garcia-Pino A, Christensen-Dalsgaard M, Wyns L, Yarmolinsky M, Magnuson RD, et al. (2008) Doc of prophage P1 is inhibited by its antitoxin partner Phd through fold complementation. J Biol Chem 283: 30821-30827.

33. Kamada K, Hanaoka F, Burley SK (2003) Crystal structure of the MazE/MazF complex: molecular bases of antidote-toxin recognition. Mol Cell 11: 875-884.

34. Takagi H, Kakuta Y, Okada T, Yao M, Tanaka I, et al. (2005) Crystal structure of archaeal toxin-antitoxin RelE-RelB complex with implications for toxin activity and antitoxin effects. Nat Struct Mol Biol 12: 327-331.

35. Dienemann C, Boggild A, Winther KS, Gerdes K, Brodersen DE (2011) Crystal structure of the VapBC toxin-antitoxin complex from Shigella flexneri reveals a hetero-octameric DNA-binding assembly. J Mol Biol 414: 713-722.
36. Kamada K, Hanaoka F (2005) Conformational change in the catalytic site of the ribonuclease YoeB toxin by YefM antitoxin. Mol Cell 19: 497-509.

37. Mattison K, Wilbur JS, So M, Brennan RG (2006) Structure of FitAB from Neisseria gonorrhoeae bound to DNA reveals a tetramer of toxin-antitoxin heterodimers containing pin domains and ribbon-helix-helix motifs. J Biol Chem 281: 37942-37951.

38. Christensen SK, Mikkelsen M, Pedersen K, Gerdes K (2001) RelE, a global inhibitor of translation, is activated during nutritional stress. Proc Natl Acad Sci U S A 98: 14328-14333.

39. Overgaard M, Borch J, Gerdes K (2009) RelB and RelE of Escherichia coli form a tight complex that represses transcription via the ribbon-helix-helix motif in RelB. J Mol Biol 394: 183-196.

40. Charette MF, Henderson GW, Markovitz A (1981) ATP hydrolysis-dependent protease activity of the lon (capR) protein of Escherichia coli K-12. Proc Natl Acad Sci U S A 78: 4728-4732.

41. Evdokimov A, Voznesensky I, Fennell K, Anderson M, Smith JF, et al. (2009) New kinase regulation mechanism found in HipBA: a bacterial persistence switch. Acta Crystallogr D Biol Crystallogr 65: 875-879.

42. Tsilibaris V, Maenhaut-Michel G, Van Melderen L (2006) Biological roles of the Lon ATP-dependent protease. Res Microbiol 157: 701-713.

43. Van Melderen L, Aertsen A (2009) Regulation and quality control by Londependent proteolysis. Res Microbiol 160: 645-651.

44. Kuroda A, Nomura K, Ohtomo R, Kato J, Ikeda T, et al. (2001) Role of inorganic polyphosphate in promoting ribosomal protein degradation by the Lon protease in E. coli. Science 293: 705-708.

45. Nomura K, Kato J, Takiguchi N, Ohtake H, Kuroda A (2004) Effects of inorganic polyphosphate on the proteolytic and DNA-binding activities of Lon in Escherichia coli. J Biol Chem 279: 34406-34410.

46. Rotem E, Loinger A, Ronin I, Levin-Reisman I, Gabay C, et al. (2010) Regulation of phenotypic variability by a threshold-based mechanism underlies bacterial persistence. Proc Natl Acad Sci U S A 107: 12541-12546.

47. Langklotz S, Narberhaus F (2011) The Escherichia coli replication inhibitor CspD is subject to growth-regulated degradation by the Lon protease. Mol Microbiol 80: 1313-1325.

48. Kim Y, Wang X, Zhang XS, Grigoriu S, Page R, et al. (2010) Escherichia coli toxin/antitoxin pair MqsR/MqsA regulate toxin CspD. Environ Microbiol 12: 1105-1121.

49. Wagner S, Baars L, Ytterberg AJ, Klussmeier A, Wagner CS, et al. (2007) Consequences of membrane protein overexpression in Escherichia coli. Mol Cell Proteomics 6: 1527-1550.

50. Datsenko KA, Wanner BL (2000) One-step inactivation of chromosomal genes in Escherichia coli K-12 using PCR products. Proc Natl Acad Sci U S A 97: 66406645 .

51. Pruteanu M, Neher SB, Baker TA (2007) Ligand-controlled proteolysis of the Escherichia coli transcriptional regulator ZntR. J Bacteriol 189: 3017-3025.

52. Lundblad JR, Laurance M, Goodman RH (1996) Fluorescence polarization analysis of protein-DNA and protein-protein interactions. Mol Endocrinol 10: 607-612.

53. Blattner FR, Plunkett G, 3rd, Bloch CA, Perna NT, Burland V, et al. (1997) The complete genome sequence of Escherichia coli K-12. Science 277: 1453-1474.

54. Haldimann A, Wanner BL (2001) Conditional-replication, integration, excision, and retrieval plasmid-host systems for gene structure-function studies of bacteria. J Bacteriol 183: 6384-6393.

55. Baba T, Ara T, Hasegawa M, Takai Y, Okumura Y, et al. (2006) Construction of Escherichia coli K-12 in-frame, single-gene knockout mutants: the Keio collection. Mol Syst Biol 2: 2006.0008.

56. Kitagawa M, Ara T, Arifuzzaman M, Ioka-Nakamichi T, Inamoto E, et al. (2005) Complete set of ORF clones of Escherichia coli ASKA library (a complete set of $\mathrm{E}$. coli K-12 ORF archive): unique resources for biological research. DNA Res 12: 291-299.

57. Ojangu EL, Tover A, Teras R, Kivisaar M (2000) Effects of combination of different -10 hexamers and downstream sequences on stationary-phase-specific sigma factor sigma(S)-dependent transcription in Pseudomonas putida. J Bacteriol 182: 6707-6713.

58. Guzman LM, Belin D, Carson MJ, Beckwith J (1995) Tight regulation, modulation, and high-level expression by vectors containing the arabinose PBAD promoter. J Bacteriol 177: 4121-4130.

59. Zaslaver A, Mayo AE, Rosenberg R, Bashkin P, Sberro H, et al. (2004) Just-intime transcription program in metabolic pathways. Nat Genet 36: 486-491. 